\title{
Simulação em saúde: história e conceitos cognitivos aplicados
}

\author{
Health simulation: history and \\ applied cognitive concepts
}

\section{Danielle Rachel dos Santos Carvalho' ${ }^{1}$ Nathalia de Moraes Lébeis Nery ${ }^{2}$ (1) Thiago Martins Santos ${ }^{3}$ (i) Dario Cecilio-Fernandes ${ }^{4}$ (1)}

\footnotetext{
${ }^{1}$ Autora para correspondência. Universidade Estadual de Campinas (Campinas). São Paulo, Brasil. drss.farmaceutica@gmail.com Universidade Estadual de Campinas (Campinas). Faculdade São Leopoldo Mandic (Araras). São Paulo, Brasil. nathalialebeis@yahoo.com.br 3-4Universidade Estadual de Campinas (Campinas). São Paulo, Brasil.santosth@unicamp.br, dario.fernandes@gmail.com
}

\section{Introdução}

A simulação é utilizada como ferramenta ou metodologia de educação há muito tempo. Apesar do aumento da utilização em diferentes áreas, como aviação e área da saúde, o seu uso muitas vezes é intuitivo. Nesse artigo, iremos discutir o conceito de simulação com uma breve revisão histórica e apresentar alguns conceitos baseados em evidência. Como o uso de simulação é extenso e em diversas áreas, iremos focar na literatura da área da saúde.

Neste artigo, utilizaremos o conceito de simulação proposto por Gaba: "a simulação é uma técnica, e não uma tecnologia, que visa substituir ou amplificar experiências reais com experiências guiadas que evocam ou replicam aspectos substanciais do mundo real em um modo totalmente interativo."1 O conceito de Gaba ${ }^{1}$ traz dois aspectos relevantes da simulação: 1) nem sempre há a necessidade de ter uma tecnologia associada ao emprego da técnica; e 2) a importância de oferecer aos participantes a possibilidade de imersão em uma tarefa ou ambiente, o que permite uma experiência mais próxima do real.
Nos cursos da área da saúde, durante a prática clínica em ambiente real, não existe a garantia de oportunidade igual para todos os alunos praticarem as habilidades propostas e nem de repetição quantas vezes forem necessárias para adquirir as habilidades treinadas ${ }^{2}$, uma vez que depende dos pacientes e dos locais em que se encontram. Já no ambiente de simulação, é possível praticar determinada habilidade quantas vezes forem necessárias até alcançar a proficiência, sem haver a preocupação de incomodar o paciente, uma vez que a prática ocorre em manequins próprios para esta ação. Além disso, pode-se oferecer diferentes situações com complexidades variadas, de acordo com os objetivos de aprendizagem e etapa da formação do estudante, para que todos tenham a oportunidade de praticar igualmente. Quando falamos em "complexidade", nos referimos à dificuldade e ao aprofundamento exigido ao caso clínico abordado ${ }^{3}$, ou seja, um cenário de parada cardiorrespiratória é considerado de alta complexidade por exigir maior conhecimento clínico e de procedimentos do que, por exemplo, um cenário de administração de medicamentos via subcutânea, considerado de baixa complexidade. 
Ultimamente, também tem se questionado os aspectos éticos e riscos do ensino no ambiente real, sem antes ter algum tipo de treinamento. A simulação possibilita aos estudantes vivenciarem problemas autênticos, em um ambiente seguro e pedagógico, tendo a oportunidade de praticar suas habilidades de forma simulada, antes de realizá-los em pacientes reais.., 5 Ou seja, durante a simulação os estudantes experimentam situações desafiadoras em um ambiente seguro e psicologicamente adequado, onde o erro é permitido, pois uma das finalidades é aprender com os erros, já que não há prejuízo aos pacientes e profissionais. ${ }^{6}$

Por meio da simulação é possível realizar o ensino e o treino em saúde, tanto de habilidades técnicas e manejo de pacientes, como também em competências relacionadas à segurança do paciente e trabalho em equipe. $\underline{7}$ Ou seja, o emprego da simulação permite criar atividades de acordo com a necessidade dos participantes, através de experiências, seguidas de um momento de reflexão guiada sobre o desempenho do participante na atividade, o debriefing, que tem impacto direto no conhecimento teórico do estudante e na sua prática profissional.. -10

\section{Retrospectiva histórica da Simulação}

Na área da saúde, a utilização da simulação é antiga, com relatos de modelos anatômicos desde a antiguidade, e uso de animais no treinamento de habilidades cirúrgicas. ${ }^{11}$ Já o uso de manequins existe há quase meio século, quando estes começaram a ser utilizados para treinamento de ressuscitação. ${ }^{12,13} \mathrm{~A}$ simulação moderna iniciou-se por um fabricante de brinquedos, o norueguês Asmund Laerdal, criador do primeiro manequim de ressuscitação, a "Resusci Annie"14 e por Denson e Abrahamson, criadores do "SimOne", que apesar de pioneiro, não conseguiu ser difundido, entre outros fatores, pelo seu alto custo na época de sua criação. ${ }^{15,16}$

Duas grandes áreas influenciaram de forma positiva a simulação na área da saúde como conhecemos hoje: os militares e a indústria da aviação. Dos militares surgiu o conceito de debriefing de grupo durante a Segunda Guerra Mundial no trabalho de S.L.A. Marshall. ${ }^{17}$ Marshall observou que quando uma pessoa descreve o que aconteceu com ela durante uma experiência angustiante permite que os colegas corrijam as percepções equivocadas e prestem apoio social, diminuindo o desenvolvimento de reações pós-traumáticas, restaurando a coesão da unidade e possibilitando o retorno ao combate. Da indústria de aviação veio a influência do realismo, ou seja, o quão real a simulação é da vida real. Tal realismo foi alcançado por meio dos simuladores de voo que surgiram a partir de 1929, o "Blue Box" ou "Link Trainer", sendo o conceito incorporado pela área da saúde. ${ }^{18}$

Um marco da simulação na área da saúde foi a publicação, em 1999, do livro "To err is human: building a safer health system" do Instituto de Medicina dos EUA, que apresentou dados de que o erro humano é a principal causa de eventos adversos que levam a desfechos desfavoráveis aos pacientes. Este estudo afirmou que os erros podem ser evitados e para isso, na área da saúde, é importante projetar sistematicamente a segurança nos processos de atendimento. O relatório, além de examinar a qualidade dos cuidados de saúde na América, apresenta uma série de recomendações para alcançar uma mudança de limiar na qualidade, e dentre elas recomenda a adoção da simulação como método para treinamento multidisciplinar. $\frac{19}{9}$

Na última década, houve um crescimento exponencial no uso de simulação na área da saúde, tanto para estudantes e residentes quanto para formação continuada de profissionais. ${ }^{20}$ A utilização deste e de outros métodos de ensino inovadores tornou-se uma necessidade devido ao mau desempenho da maioria dos graduados principalmente na prática de habilidades clínicas, aplicação de conhecimentos e resolução de problemas. ${ }^{21}$ Deve-se considerar ainda a falta de eventos críticos que sejam fonte de aprendizado e aquisição de diferentes habilidades, bem como a dificuldade em ensinar utilizando pacientes reais, garantindo seus direitos éticos e legais..22 Estes são outros fatores que privilegiaram o crescimento da utilização da metodologia baseada em simulação na área da saúde.

\section{A simulação como metodologia de ensino}

A aprendizagem baseada em simulação é caracterizada por estratégias pedagógicas, tecnologia, complexidade e amplitude de tarefas que preparam os estudantes para a vida profissional. ${ }^{23}$ Há inúmeras vantagens em utilizar a simulação como metodologia de ensino, como por exemplo: a possibilidade de repetição, de experimentar situações clínicas autênticas, 
a prática em ambiente seguro, o aprendizado com o erro, a vivência de experiências padronizadas e o feedback sobre a prática. Antes de explicarmos cada um desses conceitos, vamos abordar as questões cognitivas que envolvem a aprendizagem.

O uso da simulação melhora a aquisição de habilidades, porém estudos demonstram que essas habilidades podem decair quando não utilizadas. Por exemplo, uma habilidade cirúrgica decairá com o tempo após períodos de não uso ${ }^{24}$, sendo que a falta da habilidade necessária será uma ameaça à segurança do paciente. Estudos revelaram que estudantes não foram capazes de realizar com proficiência a habilidade necessária 125, 626,27, ou 12 26 meses após o término do treinamento. McKenna e Glendon 28 também descobriram que apenas um quarto dos 120 socorristas profissionais ainda eram proficientes 6 meses após receberem treinamento em ressuscitação cardiopulmonar.

\section{Aprendizagem na perspectiva cognitiva}

Para entendermos melhor a razão da falta de proficiência depois do treinamento, é necessário entendermos como o processo de aquisição de habilidades funciona. A psicologia cognitiva distingue conhecimento entre declarativo e procedural, onde conhecimento declarativo refere-se a "saber o quê", isto é, conhecimento sobre fatos e conhecimento procedural se refere a "saber como"..29 Enquanto a memória declarativa decai com o passar do tempo, a memória procedural não é afetada pelo tempo..$^{30}$

Toda a aprendizagem começa pelo conhecimento declarativo, por exemplo, por meio de instruções ou observações. Nesse primeiro momento, onde a maioria do conhecimento está no formato declarativo, a performance é demorada e está sujeita a erros, haja vista que o conhecimento declarativo é passível de esquecimento. O conhecimento na memória declarativa degrada com a falta de uso, levando à incapacidade de executar a tarefa. Uma falha de memória é um estado em que itens de memória declarativa necessários para executar a tarefa não podem ser recuperados da memória devido à falta de prática. No segundo momento, o conhecimento é representado usando uma mistura de memória declarativa e procedural, portanto, nesta etapa, é necessário treinamento para manter ativo o conhecimento declarativo.
No terceiro momento, o conhecimento está no formato procedural, a memória procedural é basicamente imune à deterioração. ${ }^{30}$

Desta forma, o fato de o conhecimento médico aprendido ser frequentemente esquecido, refere-se ao gerenciamento dos dois tipos de conhecimento, declarativo e procedural. Isso levanta a questão importante de que além do uso da simulação para a aprendizagem, é necessário refletir sobre como o próprio processo educacional pode ser adaptado para mudar o aprendizado para a memória de curto prazo para de longo prazo. ${ }^{31}$ Pensando em dois cenários comuns no ensino: aferição de pressão arterial e atendimento de parada cardiorrespiratória, o primeiro é uma habilidade que os alunos irão utilizar com mais frequência tanto ao longo do curso como na prática profissional, enquanto o segundo irão utilizar com pouca frequência. Por isso, não é recomendado que seja utilizado a mesma estratégia de simulação durante o ensino dos mesmos. Antes de apresentar as recomendações para os dois cenários, serão abordados alguns conceitos importantes que promovem a retenção de informações a longo prazo, como o efeito do espaçamento, da prática intercalada, da testagem e do feedback.

\section{O efeito do espaçamento}

O efeito do espaçamento ou prática espaçada refere-se à descoberta de que encontros educacionais e/ ou sessões de treinamento espaçados e repetidos ao longo do tempo resultam em uma melhor retenção de aprendizado, em comparação do ensino em um único encontro. $\frac{31}{1}$ Os benefícios de espaçar as sessões de prática estão há muito tempo confirmados na literatura, inclusive com estudos sobre a retenção de habilidades na área da saúde. Por exemplo, Spruit e colaboradores ${ }^{32}$ comprovaram que a prática espaçada no treinamento de habilidades motoras laparoscópicas facilita a aquisição de habilidades, além da retenção de curto e longo prazo sendo, portanto, mais eficiente para os estudantes.

O porquê da prática espaçada ser mais eficaz do que a prática intensiva pode ser explicada pelo fato de que para incorporar a nova aprendizagem na memória de longo prazo é necessário um processo de consolidação, ou seja, as representações da nova 
aprendizagem ganham significado, sendo fortalecidos e conectados aos conhecimentos prévios. Este processo pode demorar várias horas ou vários dias. Por isso, a aprendizagem duradoura requer tempo para as repetições mentais e os outros processos de consolidação. Quando se realiza a prática intensiva, em um único encontro, a aprendizagem apoia-se na memória de curto prazo. $\frac{33}{3}$

\section{Efeito da testagem}

O efeito de teste refere-se à vantagem de retenção de material testado em relação ao material apresentado para estudo adicional. ${ }^{34,35}$ Diversos estudos apontam que o próprio teste melhora o aprendizado e a retenção a longo prazo, sendo que testar de novo é mais eficiente do que estudar de novo. ${ }^{36,37}$ Recuperar informações da própria memória favorece a modificação do próprio traço de memória, aumentando, consequentemente, a possibilidade de sucesso futuro na recuperação das informações aprendidas. 38 Diversos estudos elucidam que o próprio teste, na forma de quizzes, jogos, flashcards, entre outros, melhora o aprendizado e a retenção a longo prazo, sendo que testar várias vezes em sessões espaçadas é mais eficiente do que estudar novamente. ${ }^{37-39}$ Estudos realizados por um mesmo grupo e utilizando a habilidade Reanimação Cardiopulmonar constataram que os alunos tiveram melhor retenção do conhecimento quando foram testados ao invés de praticar novamente a mesma habilidade. $\underline{35,39}$

\section{Prática Intercalada}

O conceito de prática intercalada refere-se à aprendizagem de duas ou mais habilidades, tópicos, conceitos ou princípios relacionados entre si de forma intercalada, uma vez que eles são misturados em vez de separados em blocos, praticando de modo mais aleatória e menos previsível. $\stackrel{40}{ } \mathrm{O}$ benefício da prática intercalada na aquisição e retenção de habilidades motoras também já é bem documentado na literatura. ${ }^{41-44}$ Porém, ainda são escassos os estudos relacionados à área da saúde.

Uma das explicações do porquê da prática intercalada ser mais eficaz do que a prática de um único conceito é que a intercalação melhora a capacidade do cérebro de distinguir ou discriminar conceitos.
Ou seja, cada tentativa de prática é diferente da última, então as respostas mecânicas não funcionam. Em vez disso, o cérebro deve se concentrar continuamente na busca de soluções diferentes. Esse processo pode melhorar sua capacidade de aprender recursos essenciais de habilidades e conceitos, 0 que permite melhor selecionar e executar a resposta correta. ${ }^{45}$ Outra explicação possível é que a intercalação permite um contexto de treinamento mais variado que a prática de um único conceito, e essa variabilidade introduz interferência, sendo benéfica para a retenção a longo prazo. $\frac{46}{}$

\section{Reflexão}

Uma outra forma de prática de recuperar informações é a reflexão. A reflexão nos ajuda a analisar o que foi aprendido em uma experiência e é considerada tão essencial quanto questionários e testes, podendo ser realizada pelos estudantes ao responderem simples perguntas, como: O que aconteceu? O que eu fiz? Como funcionou? O que eu faria diferente na próxima vez? ${ }^{33}$ Ao responder essas simples perguntas, que normalmente estão presentes na etapa final dos cenários de simulação, o debriefing, os estudantes aprendem com a experiência e podem obter melhores resultados.

\section{Feedback}

Além disso, o feedback deve estar sempre presente nas sessões de aprendizagem. De acordo com Hattie ${ }^{47}$, o feedback é uma das influências mais poderosas no aprendizado e pode ser descrito como uma informação fornecida por um agente sobre aspectos de seu desempenho ou compreensão. O feedback é fundamental para apoiar o desenvolvimento cognitivo, técnico e profissional. $\underline{48}$ Para ser efetivo, o feedback deve ser claro, objetivo, significativo, compatível com o conhecimento prévio dos alunos e fornecer conexões lógicas, promovendo um desenvolvimento positivo e desejável. ${ }^{47,48}$ É possível encontrar diversos tipos, estruturas e o momento ideal de fornecer feedback ao estudante descritos na literatura. $.8-52$

Quanto ao tipo, o feedback pode ser diretivo ou facilitador, o primeiro informa ao aluno o que requer correção, o segundo envolve o fornecimento de comentários e 
sugestões para facilitar os destinatários em sua própria revisão. Quer seja diretivo ou facilitador, o feedback também pode variar em sua estrutura: de verificação ou elaboração. O feedback de verificação indica se a resposta está certa ou errada, e de elaboração facilita o destinatário para chegar à resposta correta. Além de ser de vários tipos, o feedback pode ser estruturado de maneira diferente: pode ser escrito, verbal ou numérico. Seu formato provavelmente está relacionado ao contexto em que é gerado. A facilitação específica de resposta do feedback por escrito, gerado a partir de um exame escrito, pode aumentar sua eficácia.

O momento do feedback pode influenciar de forma independente sua eficácia. As evidências parecem sugerir que a eficácia e o momento estão relacionados ao foco e à dificuldade da tarefa. ${ }^{24} \mathrm{O}$ feedback após a simulação (postergado) pode ser melhor para apoiar a transferência de conhecimento, enquanto o feedback durante a simulação (imediato) pode ser mais eficaz em curto prazo e para apoiar o desenvolvimento de habilidades procedimentais. $\frac{24}{\mathrm{Na}}$ aprendizagem motora, que é trabalhada na simulação, a literatura recomenda utilizar o feedback postergado, que apesar de ser mais incômodo para o aprendiz, se mostra mais eficaz na aquisição de uma habilidade motora, quando comparado com o feedback imediato. Isso porque em aprendizagens motoras, uma teoria sustenta que quando o feedback é imediato, ele se torna parte da tarefa e, mais tarde, em um contexto do mundo real, sua ausência se torna uma lacuna no automatismo estabelecido, atrapalhando a performance no longo prazo. Desta forma, sugere-se a retirada gradual do feedback quando empregado durante a simulação. Vale mencionar ainda que o feedback postergado tem melhor resultado para a retenção do conhecimento.

Contudo, independente do modelo, o que defendemos é que o feedback sempre seja aplicado de maneira objetiva, permitindo que o estudante alinhe sua apreciação sobre o que ele sabe e não sabe, eliminando ilusões, de modo que sua apreciação seja ajustada para refletir melhor a realidade. $\frac{33}{\mathrm{E}} \mathrm{Em}$ simulação, os momentos de feedback são de extrema importância, pois permitem que o aluno reflita sobre o seu próprio desempenho. Refletir sobre o que deu certo, o que deu errado e como é possível fazer de modo diferente na próxima vez nos ajuda a isolar as ideias chave, organizá-las em modelos mentais e aplicá-las novamente no futuro, com vistas a aprimorar e evoluir com base no que já aprendemos. $\frac{53}{3}$
Quando falamos em aprendizagem baseada em simulação, sabemos que esta é considerada uma parte essencial da aprendizagem prática, uma vez que comprovadamente é capaz de realizar mudanças na educação e na formação, melhorando a qualidade e o desempenho dos participantes. ${ }^{21,22}$ Porém, é importante que além de nossas sessões de aprendizagem permitirem aos estudantes adquirirem os conhecimentos e habilidades essenciais para a prática profissional, elas devem garantir que os participantes sejam capazes de mobilizar os recursos necessários da memória, quando os mesmos precisarem ser aplicados em contextos futuros.

Por isso, quando alinhamos o uso da metodologia de simulação com as estratégias descritas acima, a aprendizagem se torna mais profunda e duradoura. Porém, gostaríamos de reforçar que apesar de todos os benefícios do aprendizado com a metodologia de simulação, para os estudantes atingirem a expertise em determinado assunto após o treinamento simulado, é necessário que eles vivenciem experiências clínicas com prática em ambiente real, sempre acompanhados de um supervisor e recebendo feedbacks contínuos.

\section{Recomendações}

Antes das recomendações, precisamos ver a diferença entre aquisição e retenção. Enquanto a aquisição de habilidades significa "ganhar uma nova" habilidade que não era previamente compreendida ou não existia, a retenção de habilidade se refere ao processo de desempenho após um período de não uso. A diferença entre aquisição e retenção de habilidades nos ajudará a otimizar o projeto do treinamento de simulação usando conceitos de psicologia cognitiva. Abaixo estão dois exemplos práticos de como aplicar cada um desses conceitos de acordo com seu propósito.

1. Aferição de pressão arterial: Nesse caso, o foco é em aquisição do conhecimento, pois como é uma prática que será utilizada com frequência durante a formação e na prática profissional, dificilmente o conhecimento decairá com o passar do tempo. Para situações em que o foco é aquisição de conhecimentos, o recomendado é realizar sessão única, idealmente um ou dois dias antes da prática. 
2. Atendimento de parada cardiorrespiratória: Nesse caso, o foco é em retenção do conhecimento, pois como é uma prática essencial que será pouco realizada durante a formação, o conhecimento decairá facilmente com o passar do tempo. Para situações em que o foco é retenção de conhecimentos, o recomendado é realizar sessão espaçada e intercalada, utilizando o efeito da testagem.

Sobre o feedback, se a aprendizagem possuir característica complexa, ele pode ser aplicado durante a simulação; no entanto, recomendamos que seja retirado de forma gradual. Já quando a aprendizagem tem característica mais simples, recomendamos o feedback postergado, pois o feedback imediato parece ser desfavorável nestes casos, como explicado anteriormente.

\section{Conclusão}

Neste artigo discutimos os conceitos de aquisição e retenção de conhecimentos, e como eles são importantes para a formação dos profissionais da área da saúde. Reforçamos que tanto a aquisição quanto a retenção são processos ativos, ou seja, o aprendizado ocorrerá somente se o aluno assumir um papel ativo no processo. Portanto, o uso da simulação, que é uma metodologia ativa comprovadamente eficaz, pode ter ainda melhores resultados para o aprendizado efetivo do aluno quando associada às estratégias da psicologia cognitiva.

\section{Agradecimentos}

Esta pesquisa foi parcialmente financiada pela FAPESP - Fundação de Amparo à Pesquisa do Estado de São Paulo [Projeto Jovem Pesquisador $n^{\circ}$ 2018/15642-1e Projeto Regular $n^{\circ}$ 2020/04909-7] concedida a Dario Cecilio-Fernandes e [Bolsa de Pós-Doutorado $n^{\circ}$ 2020/07132-3] concedida a Danielle Rachel dos Santos Carvalho. O financiador não teve nenhuma influência no desenho do estudo, coleta e análise de dados, decisão de publicar ou preparação do manuscrito.

\section{Referências}

1. Gaba DM. The future vision of simulation in health care. Qual Saf Health Care. 2004;13(Suppl 1):i2-10. http://dx.doi.org/10.1136/ gshc. 2004.009878
2. Vázquez-Mata G, Guillamet-LLoveras A. El entrenamiento basado en la simulación como innovación imprescindible en la formación médica. Educ Med [Internet]. 2009;12(3):149-55. Disponível em: https://scielo.isciii.es/pdf/edu/v12n3/revision.pdf

3. Brandão CFS, Collares CF, Cecilio-Fernandes D. Simuladores, pacientes padronizados e híbridos. In: Scalabrini Neto A, Fonseca AS, Brandão CFS. Simulação clínica e Habilidades na saúde. São Paulo: Editora Atheneu; 2020. pp.11-21.

4. Cook DA. How much evidence does it take? A cumulative metaanalysis of outcomes of simulation-based education. Med Educ. 2014;48(8):750-60. https://doi.org/10.1111/medu.12473

5. Wang EE. Simulation and adult learning. Dis Mon. 2011;57(11):664-78. https://doi.org/10.1016/j. disamonth.2011.08.017

6. Argullós JLP, Sancho CG. El uso de las simulaciones em educacíon médica. TESI [Internet]. 2010;11(2):147-69. Disponível em: https://revistas.usal.es/index.php/eks/article/view/7075

7. Raemer DB. Team-oriented medical simulation. In: Dunn WF, editor. Simulators in critical care education and beyond. Des Plaines, IL: Society of Critical Care Medicine; 2004. pp 42-6.

8. Kaufman DM. Applying educational theory in practice. BMJ. 2003;326(7382):213-6. https://doi.org/10.1136/bmj.326.7382.213

9. Issenberg SB, McGaghie WC, Petrusa ER, Lee Gordon D, Scalese RJ. Features and uses of high-fidelity medical simulations that lead to effective learning: a BEME systematic review. Med Teach. 2005;27(1):10-28. https://doi.org/10.1080/01421590500046924

10. Zigmont JJ, Kappus LJ, Sudikoff SN. The 3D model of debriefing: defusing, discovering, and deepening. Semin Perinatol. 2011;35(2):52-8. https://doi.org/10.1053/j.semperi.2011.01.003

11. Neri-Vela R. El origen del uso de simuladores en Medicina. Rev Fac Med UNAM [Internet]. 2017;60(S1):21-7. Disponível em: https://www.medigraphic.com/cgi-bin/new/resumen. cgi?IDARTICULO=77104

12. Perkins GD. Simulation in resuscitation training. Resuscitation. 2007;73(2):202-11. https://doi.org/10.1016/j. resuscitation.2007.01.005

13. Sahu S, Lata I. Simulation in resuscitation teaching and training, an evidence-based practice review. J Emerg Trauma Shock. 2010;3(4):378-84. https://doi.org/10.4103/0974-2700.70758

14. Tjomsland N, Baskett $P$, Laerdal AS. Resuscitation. 2002;53(2):115-9. https://doi.org/10.1016/s0300-9572(02)00033-3

15. Denson JS, Abrahamson S. A computer-controlled patient simulator. JAMA. 1969;208(3):504-8. Citado em: PMID: 5818529

16. Rosen KR. The history of medical simulation. J Crit Care. 2008;23(2):157-66. https://doi.org/10.1016/j.jcrc.2007.12.004 
17. Marshall SLA. Bringing up the Rear: A Memoir. San Rafael, US: Presidio Press; 1979.

18. Bradley P. The history of simulation in medical education and possible future directions. Med Educ. 2006;40(3):254-62. https:// doi.org/10.1111/j.1365-2929.2006.02394.x

19. Kohn LT, Corrigan J, Donaldson MS. To err is human: building a safer health system. Washington, D.C.: National Academy Press; 2000.

20. Kelly M, Jeffries P. Clinical simulation in health care-Contemporary learning for safety and practice. Collegian. 2012;19(3):115-6. https://doi.org/10.1016/j.colegn.2012.07.002

21. Elshama SS. How to Develop Medical Education (Implementation View). Atlanta: Scholars' Press; 2016.

22. McGaghie WC, Issenberg SB, Petrusa ER, Scalese RJ. A critical review of simulation-based medical education research: 20032009. Med Educ. 2010;44(1):50-63. https://doi.org/10.1111/j.13652923.2009.03547.x

23. Elshama SS. How to apply Simulation-Based Learning in Medical Education? Iberoam J Med. 2020;2(2):79-86. http://doi. org/10.5281/zenodo.3685233

24. Cecilio-Fernandes D, Cnossen F, Jaarsma DA, Tio RA. Avoiding Surgical Skill Decay: A Systematic Review on the Spacing of Training Sessions. J Surg Educ. 2017;75(2):471-80. https://doi. org/10.1016/j.jsurg.2017.08.002

25. Moazed F, Cohen ER, Furiasse N, Singer B, Corbridge TC, McGaghie WC, et al. Retention of critical care skills after simulation-based mastery learning. J Grad Med Educ. 2013;5(3):458-63. https://doi.org/10.4300/jgme-d-13-00033.1

26. Barsuk JH, Cohen ER, McGaghie WC, Wayne DB. Long-term retention of central venous catheter inser- tion skills after simulation-based mastery learning. Acad Med. 2010;85(10 suppl):S9-12. https://doi.org/10.1097/acm.0b013e3181ed436c

27. Wik L, Myklebust H, Auestad BH, Steen PA. Retention of basic life support skills 6 months after training with an automated voice advisory manikin system without instructor involvement. Resuscitation. 2002;52(3):273-9. https://doi.org/10.1016/s03009572(01)00476-2

28. McKenna S, Glendon A. Occupational first aid training: decay in cardiopulmonary resuscitation (CPR) skills. J. Occup. Psychol. 1985;58(2):109-17. https://doi.org/10.1111/j.2044-8325.1985. $\underline{\mathrm{tb} 00186 . x}$

29. Anderson JR. Acquisition of cognitive skill. Psychol Rev. 1982;89(4):369-406. https://psycnet.apa.org/doi/10.1037/0033295X.89.4.369

30. Kim JW, Ritter FE, Koubek RJ. An integrated theory for improved skill acquisition and retention in the three stages of learning. Theor Issues Ergon Sci. 2013;14(1):22-37. https://doi. org/10.1080/1464536X.2011.573008
31. Kerfoot BP, DeWolf WC, Masser BA, Church PA, Federman $D D$. Spaced education improves the retention of clinical knowledge by medical students: a randomised controlled trial. Med Educ. 2007;41(1):23-31. https://doi.org/10.1111/j.13652929.2006.02644.x

32. Spruit EN, Guido PH, Jaap B, Hamming F. Increasing efficiency of surgical training: effects of spacing practice on skill acquisition and retention in laparoscopy training. Surg Endosc. 2015; 29(8):2235-43. https://doi.org/10.1007/s00464-014-3931-x

33. Brown $\mathrm{P}$, Roediger HL, McDaniel MA. Fixe o conhecimento: a ciência da aprendizagem bem-sucedida. Porto Alegre: Penso; 2018.

34. Allen GA, Mahler WA, Estes WK. Effects of recall tests on long-term retention of paired associates. J Verbal Learning Verbal Behav. 1969;8(4):463-70. https://psycnet.apa.org/doi/10.1016/ s0022-5371(69)80090-3

35. Kromann CB, Jensen ML, Ringsted $C$. The effect of testing on skills learning. Med Educ. 2009;43(1):21-7. https://doi.org/10.1111/ j.1365-2923.2008.03245.x

36. Agarwal P, Karpicke J, Kang S, Roediger H, McDermott K. Examining the Testing Effect with Open- and Closed-Book Tests. Appl Cogn Psychol. 2008;22(7):861-76. https://doi.org/10.1002/ acp.1391

37. Roediger HL, Karpicke JD. The Power of Testing Memory: Basic Research and Implications for Educational Practice. Perspect Psychol Sci. 2006;1(3):181-210. https://doi.org/10.1111\%2Fj.17456916.2006.00012.x

38. Eisenkraemer RE, Lilian AJ, Stein M. Uma Revisão Sistemática Sobre o Efeito de Testagem na Aprendizagem. Paidéia. 2013;23(56):397-406. https://doi.org/10.1590/1982 43272356201314

39. Kromann CB, Bohnstedt C, Jensen ML, Ringsted C. The Testing Effect on Skills Learning Might Last 6 Months. Adv Health Sci Educ. 2015;41(5):1540-7. https://doi.org/10.1007/s10459-009-9207-x

40. Benassi VA, Overson CE, Hakala CM. Applying science of learning in education: Infusing psychological science into the curriculum [Internet]. University of New Hampshire: Society for the Teaching of Psychology; 2014. Disponível em: https:// teachpsych.org/ebooks/asle2014/index.php

41. Taylor K, Rohrer D. The effects of interleaved practice. Appl Cogn Psychol. 2010;24(6):837-48. https://doi.org/10.1002/ acp.1598

42. Mayfield KH, Chase PN. The effects of cumulative practice on mathematics problem solving. J Appl Behav Anal. 2002;35(2):10523. https://dx.doi.org/10.1901\%2Fjaba.2002.35-105

43. Carpenter SK, Mueller FE. The effects of interleaving versus blocking on foreign language pronunciation learning. Memo Cognit. 2013;41:671-82. https://doi.org/10.3758/s13421-012-0291$\underline{4}$ 
44. Hall KG, Domingues DA, Cavazos R. Contextual interference effects with skilled baseball players. Percept Mot Skills. 1994;78(3 Pt 1):835-41. https://doi.org/10.2466/pms.1994.78.3.835

45. Pan SC. The interleaving effect: mixing it up boosts learning. Sci Am [Internet]. 2015;313(2). Disponível em: http://www.tinyurl. com/y49q9bnn

46. Horvath JC, Lodge JM, Hattie J, editores. From the laboratory to the classroom: Translating science of learning for teachers. Nova Iorque; Londres: Routledge; 2017.

47. Hattie J, Timperley H. The Power of Feedback. Rev Educ Res. 2007;77(1):81-112. https://doi.org/10.3102\%2F003465430298487

48. Archer JC. State of the science in health professional education: effective feedback. Med Educ. 2010;44(1):101-8. https://doi.org/10.1111/j.1365-2923.2009.03546.x
49. Waldrop PB, Justen JE, Adams TM. A Comparison of Three Types of Feedback in a Computer-Assisted Instruction Task Author(s). Education Technology [Internet]. 1986;26(11):43-5. Disponível em: https://www.jstor.org/stable/44424764

50. Jaehnig W, Miller ML. Feedback Types in Programmed Instruction: A Systematic Review. Psychol Rec. 2007;57(2):219-32. http://dx.doi.org/10.1007/BF03395573

51. Finn B, Metcalfe J. Scaffolding feedback to maximize longterm error correction. Mem. Cogn. 2010;38(7):951-61. https://doi. org/10.3758/MC.38.7.951

52. Kulik JA, Kulik CC. Timing of feedback and verbal learning. Rev Educ Res. 1988;58(1):79-97. https://doi. org/10.3102\%2F00346543058001079

53. Gernsbacher MA, Varner KR, Faust ME. Investigating differences in general comprehension skill. J Exp Psychol Learn Mem Cogn. 1990;16(3):430-45. https://doi.org/10.1037//02787393.16.3.430 